\title{
HIGH RESOLUTION RAIL-TO-RAIL ADC IN CMOS DIGITAL TECHNOLOGY
}

\author{
Daniele Gardino and Franco Maloberti
}

\author{
Department of Electronics, University of Pavia \\ Via Ferrata 1, 27100 Pavia, Italy \\ Tel. +390382 505205, Fax. +390382505677 \\ E-Mail: gardino@ele.unipv.it, franco@ele.unipv.it
}

\begin{abstract}
This paper discusses the design issues for an high resolution rail-to-rail analog-to-digital converter. The circuit proposed uses conventional digital technology (without precise capacitors) and allows 12 bits of resolution to be achieved using $2.4 \mathrm{~V}$ bias and $2.4 \mathrm{~V}$ input dynamic range. The proposed architecture is based on the successive algorithm technique and uses a rail-to-rail autozeroed comparator. A method to digitally correct errors from the first part of the conversion cycle is also discussed.
\end{abstract}

\section{INTRODUCTION}

Data converters are important interfaces for digital processing systems. The normal requirement is high resolution (more than 10 bits) to match the processing capability of digital sections. The technology normally used is optimized for digital performance; it typically provides multi-metal layers but only a single poly is available for the circuit designer. Consequently, the performance of integrated capacitors does not satisfy the accuracy required, either in terms of matching or linearity. Moreover, many systems require a large dynamic range for analog signals: often it is necessary to provide an analog dynamic range comparable with the rail voltages used.

These conditions open up new issues to ensure suitables designed data converters; the resolution must be 12 or more bits, the bandwidth in the order of $1 \mathrm{MHz}$, area and power consumption must be kept at a low level while maintaining optimized the technology for digital performances. This paper presents circuit solutions capable of properly answering the given requests. The proposed architecture employes a three step resistive divider. Moreover, it utilizes novel method for correcting digital errors occurring during the first 8 clock cycles of the successive approximation algorithm. The clock frequency foreseen is $16 \mathrm{MHz}$.

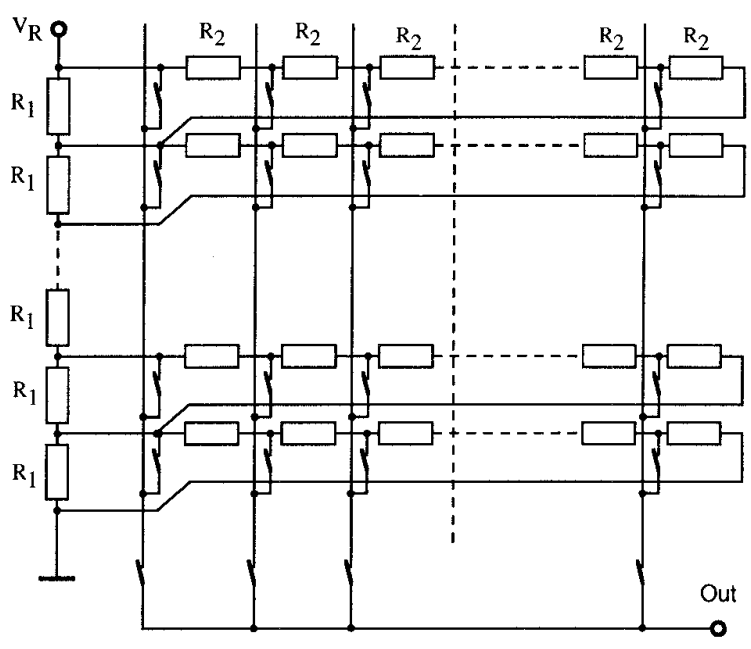

Figure 1. Conventional resistor array DAC [1]

\section{ARCHITECTURE}

The successive approximation algorithm requires the use of a DAC with the same resolution as the ADC ( 12 bits in our case). If the technology is optimized for digital applications, capacitive arrays do not allow high resolutions to be achieved because of practical and linearity limits. Metalmetal capacitors have a very low specific capacitance, thus requiring large area for array implementation. In contrast, gate-oxide capacitors have a large specific capacitance but display non linear behavior. Therefore, for resolutions as high as 12 bits resistors are necessary.

A DAC architecture based on resistors was proposed in [1]. Its schematic is shown in Fig. 1. The circuit employes a $x-y$ division of the reference voltage. The vertical resistor string performs a rough division of $\mathrm{V}_{\mathrm{R}}$; the horizontal string achieves a fine division. A suitable matrix of switches allow us to connect all the intermediate nodes to the output terminal. The performance of the architecture is suitable up to 10 


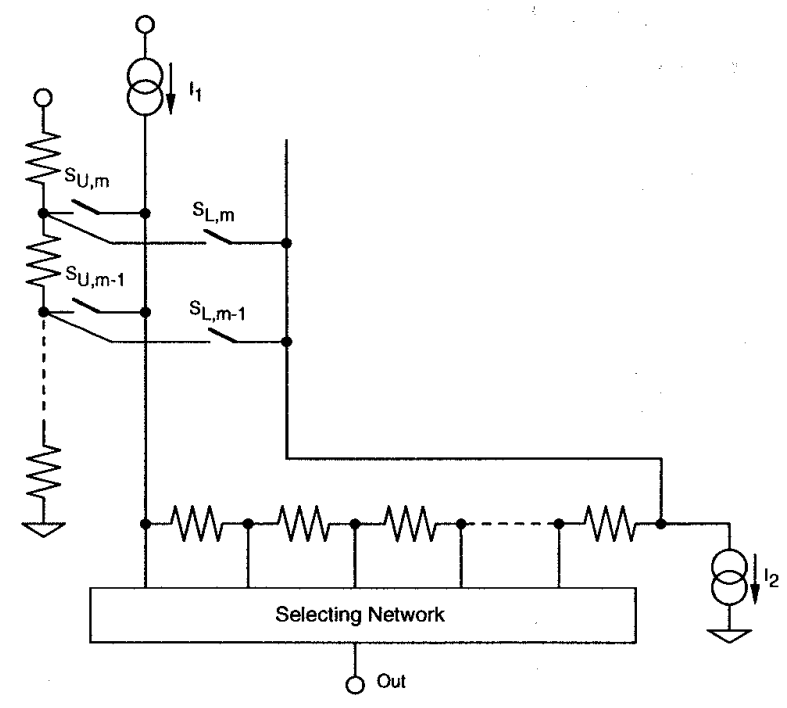

Figure 2. DAC with mobile resistor string

bits of accuracy but, unfortunately, for higher resolution the size of the $x-y$ array and speed limits coming from the equivalent $\mathrm{RC}$ time constant of the entire network make the solution impractical.

The chip area and the capacitive load to the output node is reduced by of the floating string technique [2]. The circuit, shown in Fig. 2 is a modification of the one in Fig. 1. It uses only one horizontal string that is connected in parallel to its relative coarse division. The switches $S_{U, i}$ and $S_{L, i}$ connect the floating horizontal string to the upper and the lower node of the coarse interval. Two equal current sources $I_{1}$ and $\mathrm{I}_{2}$, both nominally equal, inject a suitable current in the horizontal string, their value increases the equivalent impedance coming from the string connection [3].

The solution significantly reduces the capacitive load seen at the output node, thus allowing high speed of operation. However, the mismatch affecting currents sources and their finite output impedance limits the accuracy of the converter. The mismatch currents flowing through the parallel connection switches leads to an error that, for practical situations, limits the accuracy to 10 bits.

The solution proposed by this paper is shown in Fig. 3. It combines the advantage of the circuit in Fig. 1 and the one in Fig. 2. To achieve 12 bit resolution the architecture employes the cascade of a coarse divider, a fine divider and a super-fine divider ( $4+4+4$ bits). The first two dividers coincide with the architecture shown in Fig. 1: the main (vertical) divider comprises 16 equal resistors $R_{1}$, and, across each resistor we have the coarse series connection of 16 resistors $R_{2}$. Therefore, the voltage defined by the first two divider is $\mathrm{V}_{\mathrm{R}} / 2^{8}$. We obtain further discretization by 16 with a super-fine floating string. Instead of using resistors and

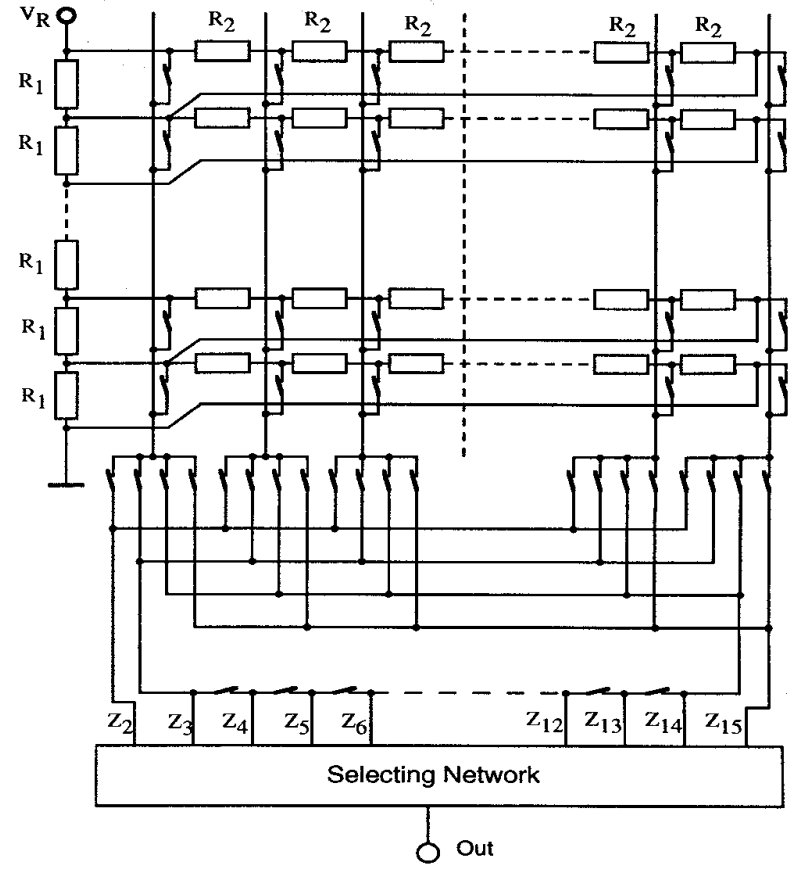

Figure 3. DAC based on resistors array and mobile $\mathrm{ON}$-switches string

balancing current sources we use using 16 on-switches. The switches used to connect the floating string operate, at the same time, as selecting elements and voltage dividers. Assuming that the reference voltage is $2.4 \mathrm{~V}$ the voltage across the switch string is about $10 \mathrm{mV}$. Therefore, the variation of the switch on-resistance is negligible and it does not affect the integral linearity. The floating string connection uses an $x-y$ logic. The switches inside the matrix select one of the rows and the switches on the bottom of the figure select the proper fine segment. Observe that we use four switches per fine selected column. From left to right they perform: measure of the voltage after the first switch $Z_{2}$, the connection to $Z_{3}$, the connection to $Z_{4}$ and measure the voltage before the last switch $\mathrm{Z}_{15}$.

The architecture in Fig. 3 has been simulated at system level and using Spice. The design target is to achieve a fast settling at the output of the DAC, assumed loaded with $1 \mathrm{pF}$ (input of the comparator). Moreover power consumption must be as low as possible (say, for $1 \mathrm{MHz}$ conversion rate, $1 \mathrm{~mW}$ ) [4]. The power consumption limits leads to $R_{1}=400$ $\Omega$; in addition we choose $R_{2}=200 \Omega$. The latter figure allows to be used as switches two complementary MOS transistors with $\mathrm{W} / \mathrm{L}=8$. The above figure allows the $\mathrm{DAC}$ to have an equivalent time constant as low as $5.68 \mathrm{nsec}$. Moreover the DAC reaches the steady state, within $0.5 \mathrm{LSB}$, in 56 nsec. The connection of the floating string of switches 


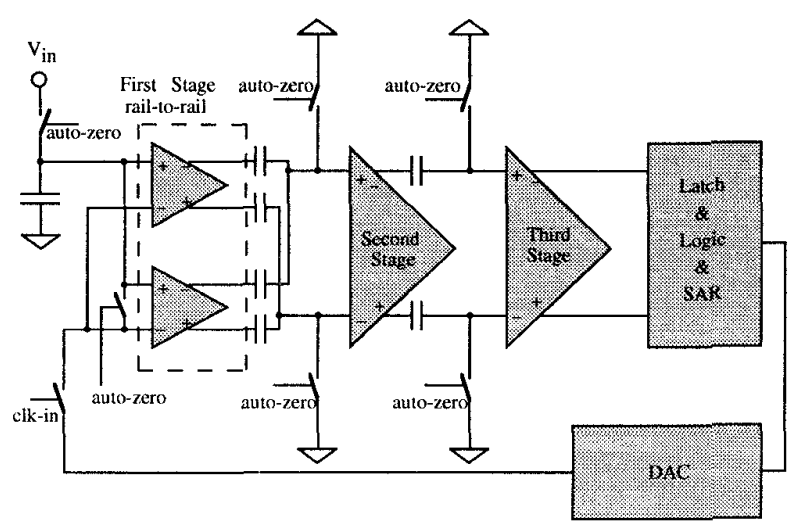

Figure 4. Successive Approximation ADC architecture

changes the voltage of the fine divider: the upper node drops a bit while the lower node goes up. In any case, for the used resistor values and transistor sizing the shift is less than \pm 0.25 LSB under the worst conditions.

As we will see shortly, the conversion requires 16 clock periods corresponding to $896 \mathrm{nsec}$ which are well below the 1 $\mathrm{MHz}$ period. Therefore, the expected conversion rate is $1 \mathrm{MS} / \mathrm{s}$. An additional advantage of the given solution is that it achieves a rail-to-rail operation: the DAC can work with $\mathrm{V}_{\mathrm{R}}=\mathrm{V}_{\mathrm{DD}}$. The comparator designed also needs a rail-to-rail input stage.

\section{COMPARATOR}

Fig. 4 shows the block diagram of the complete ADC. It includes the digital logic, the SAR and the DAC in feedback connection with the comparator. We achieve rail-to-rail operation with the input stage shown in Fig. 5 [5]. We have two complementary architectures made by a level shift and a differential stage. The resulting four output signals are processed by the second stage of amplification. In the differential stage, We avoid a common mode feedback by using, as loads, transistors in the triode region. The outputs of the two parallel input stages are combined with a capacitive adder (see Fig. 4). The capacitors used are non linear elements but the effect is not important for circuit operation.

The circuit achieves the auto-zero by shorting the inputs of the first and the second stage [6]. The inputs of the third stage are shorted as well to ensure proper biasing to the auto-zero capacitors of the second stage.

The gain of each stage is kept low $(20 \mathrm{~dB})$ to speed-up overall operation. Simulations, using a $0.5 \mu \mathrm{CMOS}$ digital technology, show that with $0.5 \mathrm{mV}$ at the input, the output reaches the level required for proper driving of the latch within 26 nsec. The power consumption of the entire comparator ( $2.4 \mathrm{~V}$ bias) is less than $0.3 \mathrm{~mW}$.

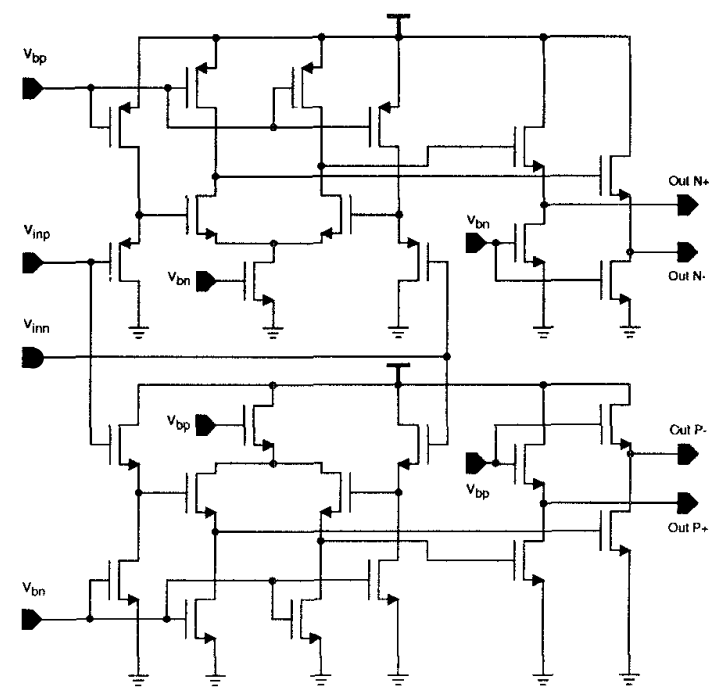

Figure 5. Rail-to-rail input stage of the comparator

\section{DIGITAL CORRECTION}

One of the main limits to circuit accuracy comes from the errors due to the comparator operation in the first part of the conversion cycle. It may happen that the input of the comparator just crosses the threshold after being driven by a large signal with opposite polarity. The recovery from a large overdrive is not fast enough and the output of the comparator can be wrong.

To correct this class of errors we use the following strategy: after $\mathrm{k}$ clock cycles, the S.A.R., instead of changing the DAC control by $V_{R} / 2^{k}$, varies the expected step by two times (double step). This simple modification of the algorithm allows errors as large as $2^{\mathrm{N}-1-\mathrm{k}} \mathrm{LSB}$ to be corrected. The cost of the technique is an additional clock cycle to achieve the same resolution. In our specific design we used $\mathrm{k}=8$; therefore, we can correct errors up to $8 \mathrm{LSB}$.

Fig. 6 shows the sequence of voltages generated by the DAC after the double step S.A.R. control. Fig. 6 a) compares conventional operation with the new solution in the case there is no previous error in the search tree. The dotted line depicts the normal operation and, as expected, the output of the DAC approaches the input voltage at the end of the conversion cycle, by $1 / 2$ LSB. The solid line shows the response with the double step. We observe that again at the end of the conversion cycle we approach the input voltage by $1 / 2$ LSB, but at the cost of one additional clock cycle. The additional clock cycle is required to compensate the double step anomaly. Fig. 6 b) shows the output of the DAC in the case of a previous error in the search tree. The dotted line shows that the error (6 LSB in the diagram) cannot be recovered. Instead the solid line shows that the double step occurring at the period \#9 allows the error to be fully recov- 




Figure 6. Error correction Algorithm

ered. In summary, the technique used is efficient and does not require much extra circuitry unlike what is required, for example, with digital correction techniques.

The limit of the method is that possible errors occurring after the bit \#9 are not corrected. However, the signals across the comparator after the clock period \#9 are quite low and this normally ensures proper operation of the circuit. Of course we can correct larger errors; the double step must be applied early in the conversion cycle. However, the errors occurring after the double step are not recovered. Therefore, a suitable trade-off between error coverage and error amplitude is necessary. An additional advantage of the proposed method is that the slewing requirements at the comparator are relaxed. Therefore, a reset phase after the comparison, normally used to neutralize the memory effect, is no longer necessary. This possibility allows us to perform the autozero only at the beginning of the conversion cycle and to use complete clock cycles for the comparison. Thus, we gain a significant speed advantage.

Fig. 7 shows the timing diagram used. The successive approximation algorithm requires the sampling of the input signal. As shown in Fig. 4, the sampling is performed during the autozero phase. We use the input capacitance of the comparator and an additional sandwitch capacitor as a storing element [7]. Since sampling accuracy depends on the input time constant, three clock periods are used for the sampling + auto-zero phase. The 12 bit conversion (with the double step phase) needs 13 clock periods. Therefore a total of 16 clock periods are necessary to perform the complete conversion.

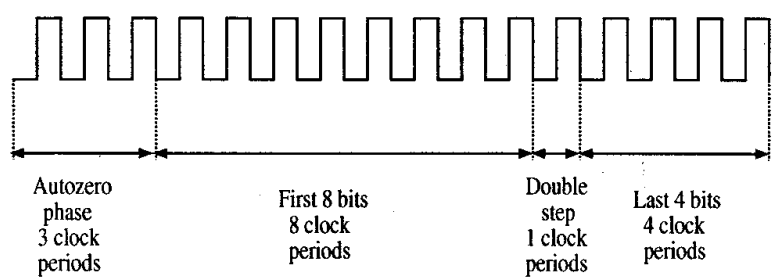

Figure 7. Timing diagram

\section{CONCLUSIONS}

This paper discussed an architecture for an ADC converter that can be integrated using CMOS digital technology. The proposed architecture properly combines the resistive $x-y$ division of a reference voltage and the floating resistive string technique. Circuit simulation show that the architecture can operates with $2.4 \mathrm{~V}$ rail and achieves 12 bit accuracy. A novel method for error correction is also discussed. It represents a good trade-off between complexity and efficiency.

\section{REFERENCES}

[1] M.J.M. Pelgrom, "A 10-b 50-MHz CMOS D/A Converter with $75 \Omega$ Buffer", IEEE Journal Solid-State Circuits, Vol. 25, pp. 1347-1352, Dec. 1990.

[2] F. Maloberti, R. Rivoir and G. Torelli, "Power Consumption optimization of 8 bit, $2 \mathrm{MHz}$ Voltage Scaling Subranging CMOS 0.5 m DAC', ICECS '96 pp. 1162-1165.

[3] B. Razavi, "Principles of Data Conversion System Design", IEEE Press pp. 79-82, 1995

[4] F. Maloberti, F. Francesconi, P. Malcovati, O.J.A.P. Nys, "Design Consideration on Low-Voltage, Low-Power Data Converter Design", IEEE Trans. Circuit and Systems - I, Vol. 42, pp. 853-863, Nov. 1995.

[5] R. Rivoir and F. Maloberti, "A $1 \mathrm{mV}$ resolution, $10 \mathrm{MS} / \mathrm{s}$ Rail-to-Rail Comparator in $0.5 \mu \mathrm{m}$ Low-Voltage CMOS Digital Process", IEEE International Symposium Conference ISCAS '97 Vol. 1, pp. 461-464, 1997.

[6] B. Razavi and B. A. Wooley, "Design Techniques for HighSpeed, High-Resolution Comparators", IEEE JSSC, Vol 7, No. 12, pp. 1916-1926, Dec 1992

[7] J.T.Wu and B.A. Wooley, "A 100-MHz Pipelined CMOS Comparator", IEEE JSSC, Vol 23, No. 6, pp. 1379-1385, Dec 1988 